\title{
Cálculo de lente intraocular en un paciente con antecedente de cirugía LASIK bilateral y colocación de implante intracorneal para presbicia
}

\section{Intraocular lens power calculation after bilateral myopic LASIK and monocular corneal inlay for presbyopia surgery}

\author{
Fidel Camacho-Cano ${ }^{1}$, Alejandro Sámano-Guerrero ${ }^{2 *}$, Filiberto Castillo-Gómez ${ }^{3}$, Arturo Enríquez-Huerta ${ }^{4}$ \\ y Rogelio Villarreal-Villarrea/ ${ }^{5}$ \\ ${ }^{1}$ Cirujano oftalmólogo, subespecialista en Cirugía Refractiva y Segmento Anterior y en Córnea y Superficie Ocular, Jefe del Departamento de \\ Cirugía Refractiva, Clínica de Ojos Monterrey; ${ }^{2}$ Médico cirujano y partero, Director General de Consultora en Investigación Analimed, Investigador \\ asociado, Fundación Ojos para México; ${ }^{3}$ Médico cirujano y partero, Investigador asociado, Clínica de Ojos Monterrey; ${ }^{4}$ Cirujano oftalmólogo, \\ Subespecialista en Vitreo y Retina, Director General, Clínica de Ojos Monterrey; ${ }^{5}$ Cirujano Oftalmólogo, Subespecialista en Cirugía Refractiva, \\ Córnea y Catarata, Profesor en Departamento de Oftalmología, Universidad de Monterrey. Monterrey, México
}

\section{Resumen}

Se presenta un paciente masculino de 55 años con antecedente de cirugía LASIK para miopía en ambos ojos, retoque en ojo izquierdo y colocación de implante intracorneal en ojo derecho para corrección de presbicia con motivo de consulta de disminución de agudeza visual. Tras diagnosticar catarata en ambos ojos se decide realizar cirugía de facoemulsificación calculando el poder del lente intraocular con la técnica afáquica de Mackool. Esta técnica se compara con el resultado de 4 equipos de evaluación corneal y 2 fórmulas de lente intraocular. Tras 2 meses de cirugía bilateral la agudeza visual lejana no corregida en ambos ojos fue de 20/25 y la cercana de J6, el error refractivo resultó dentro del rango esperado para esta técnica ( $\pm 0.75 \mathrm{dpt}$ ). La técnica afáquica de Mackool para cálculo de lente intraocular es menos práctica que las técnicas habituales, pero los resultados son aceptables y confiables. Se recomienda utilizar esta técnica en casos en los que la estructura de la córnea se encuentra alterada por dolencias o procedimientos anteriores.

Palabras clave: Implante intracorneal. Catarata. Mackool. Afaquia. Lente intraocular.

\begin{abstract}
A 55-year old male with history of bilateral LASIK surgery for myopia, left eye LASIK enhancement and right eye corneal inlay for presbyopia correction presents with diminished visual acuity. Bilateral cataract is diagnosed and phacoemulsification surgery is performed after calculating intraocular lens power with Mackool's aphakic technique. This technique was compared with the results of four corneal evaluation platforms and two intraocular lens calculation formulas. Two months after bilateral surgery, uncorrected binocular distance vision was 20/25 and uncorrected binocular near vision was J6, the postoperative refractive error was within the expected range for the aphakic technique $( \pm 0.75 \mathrm{dpt})$. The Mackool's aphakic technique is more unpractical but the results are reliable. We recommend it in these difficult cases where several surgical procedures alter the structure of the cornea.
\end{abstract}

Key words: Inlay. Cataract. Mackool. Aphakia. Intraocular len.

\section{Correspondencia:}

*Alejandro Sámano-Guerrero

E-mail: alejandrosamanog@gmail.com
Disponible en internet: 14-05-2018 Rev Mex Oftalmol. 2018;92(3):160-164 www.rmo.com.mx CC BY-NC-ND (http://creativecommons.org/licenses/by-nc-nd/4.0/). 


\section{Introducción}

La evaluación corneal tras una cirugía refractiva siempre presenta dificultades, ya sea por la medición de la curvatura corneal o la de la longitud axial. Para que los pacientes con este antecedente reciban el lente intraocular (LIO) más adecuado en una cirugía de catarata, se han desarrollado diversas fórmulas de cálculo de LIO.

La exactitud de poder de lente que requiere el paciente depende sobre todo de las medidas biométricas del ojo, la curvatura de la córnea y la confiabilidad de las fórmulas para su cálculo ${ }^{1}$. El problema principal al hacer el cálculo de poder de LIO en pacientes con antecedente de cirugía refractiva corneal es que las mediciones queratométricas varían dependiendo de la zona a estudiar en la córnea. Incluso, la cirugía refractiva altera la relación anteroposterior de la córnea, por lo que determinar el poder real de la misma puede ser un reto. Más de 20 fórmulas se han desarrollado para realizar el cálculo de LIO en este tipo de sujetos, pero ninguna ha resultado superior. Dentro de los distintos métodos de cálculo de LIO para estos pacientes existen los que requieren de datos de historias clínicas pasadas ${ }^{2}$, los que necesitan datos de historias clínicas pasadas y una topografía actual ${ }^{3}$, los que requieren de otro tipo de evaluaciones corneales actuales ${ }^{4-8}$ y los métodos que no requieren mediciones corneales 0 axiales $^{9,10}$.

Además existen otros procedimientos que igualmente pueden alterar la estructura corneal, como el cambio producido por el principio refractivo del implante intracorneal Raindrop ${ }^{\circledR}$ Near Vision Inlay (ReVision Optics, Inc., CA, EE. UU.), que presenta el paciente descrito en este reporte de caso. El lente Raindrop ${ }^{\circledR}$ obtuvo permiso para ser utilizado en EE. UU. por la FDA el día 30 de junio de 2016, por lo que se espera también pueda ser aprobado su uso en México en un futuro cercano, elevando la cantidad de casos con características similares al que describimos. El implante intracorneal Raindrop ${ }^{\circledR}$ es un lente de hidrogel transparente con el mismo índice refractivo que la córnea que se utiliza para tratamiento de presbicia. El implante Raindrop ${ }^{\circledR}$ es colocado en el estroma corneal del ojo no dominante tras la creación de un flap con femtoláser y es centrado con la ayuda de constricción pupilar. El implante Raindrop $^{\circledR}$ trabaja alterando biomecánicamente la superficie anterior de la córnea incrementando el volumen del estroma y el epitelio directamente anterior al implante, esto permite que aumente el poder dióptrico en el punto más central, mientras gradualmente ese poder se reduce en las áreas periféricas. Este mecanismo permite que el paciente pueda enfocar la visión a distancias cercanas, intermedias y lejanas. En pacientes que son aproximadamente emétropes el implante Raindrop ${ }^{\circledR}$ permite mejorar significativamente la visión cercana e intermedia sin causar cambios contraproducentes en la visión lejana binocular. Este implante permite tratar la presbicia, siendo una cirugía menos invasiva que la colocación de un LIO multifocal y al mismo tiempo con la ventaja de ser removible. Como desventaja, esta es una herramienta relativamente nueva y se desconoce de manera satisfactoria su seguridad de uso ${ }^{11,12}$.

Los pacientes con antecedente de procedimientos de cirugía refractiva sobre la córnea son pacientes que buscan soluciones independientes a la utilización de anteojos, es por eso que la cirugía de catarata en estos representa un reto. El reto es aún más grande considerando que el cirujano oftalmólogo muchas de las veces no posee la información corneal preoperatoria del paciente ni conoce el cambio refractivo que se obtuvo con estas cirugías.

Buscando desarrollar un método que pueda evadir estos obstáculos, Mackool, en el año 2006, describió una técnica afáquica para el cálculo de poder de LIO tras cirugía corneal refractiva. En este método no es necesario contar con la información queratométrica ni de la longitud axial del globo ocular. En esta técnica se ingresa el paciente a quirófano, se remueve la catarata y se realiza una refracción manifiesta al paciente media hora después; esta refracción manifiesta se utiliza para el cálculo de LIO según una fórmula diseñada por Mackool et al. Los resultados de Mackool et al. variaron dentro de $\pm 0.75 \mathrm{dpt}$ de refracción manifiesta postoperatoria $^{10}$.

\section{Presentación del caso}

Paciente masculino de 55 años de edad con antecedente de cirugía LASIK bilateral para miopía en 2004, colocación de implante intracorneal Raindrop ${ }^{\circledR}$ Near Vision Inlay en ojo derecho (OD) para corrección de presbicia en 2005 y retoque de cirugía LASIK en ojo izquierdo (OI) en 2006, acude el mes de diciembre de 2014 por disminución de agudeza visual lejana y cercana en ambos ojos. La agudeza visual lejana mejor corregida en OD fue de 20/60 en la escala de Snellen con una refracción subjetiva de $-5.50-0.75 \times 143^{\circ}$. La agudeza visual mejor corregida en Ol fue 20/80 con una refracción subjetiva de -9.00-1.25 × 42 ${ }^{\circ}$. A la exploración con lámpara de hendidura se halló un flap de cirugía LASIK en ambos ojos, una cicatriz circular en la córnea 
derecha correspondiente al implante Raindrop ${ }^{\circledR}$ (Fig. 1) y catarata bilateral (LOCSIII NO4 NC4 C1 P1). La evaluación preoperatoria incluyó el cálculo de poder de LIO utilizando 4 plataformas distintas de medición corneal: 1) un paquímetro Scheimpflug (Pentacam; Oculus, Alemania), 2) un sistema de topografía corneal (Orbscan II; Bausch \& Lomb, EE. UU.), 3) interferometría óptica de coherencia parcial (IOL Master 500; Carl Zeiss AG, Alemania) y 4) un queratómetro automatizado (Canon RK-F1 Auto Ref-Keratometer; Canon Inc., Japón). Dos fórmulas distintas para cálculo de IOL (SRK/T y Shammas) se utilizaron con los resultados de cada una de estas evaluaciones queratométricas para realizar un cálculo de LIO.

La extracción de catarata de ambos ojos se llevó a cabo previa anestesia tópica y posterior realización de facoemulsificación a través de una incisión corneal autosellante de $3 \mathrm{~mm}\left(\mathrm{OD} 60^{\circ}\right.$, Ol $\left.180^{\circ}\right)$.

La primera cirugía se realizó en el Ol. Media hora más tarde, se evaluó la refracción subjetiva a una distancia de vertex de $12 \mathrm{~mm}$. La refracción subjetiva fue de +12.00-0.50 × 126 20/50, calculándose con la fórmula de Mackool un LIO necesario de +20 dpt. El paciente regresó al quirófano, donde se le implantó el LIO Acrysoft IQ SN60WF Power (Alcon, Fort Woth, Texas, USA). La misma técnica afáquica se utilizó para el OD 3 semanas más tarde. La refracción subjetiva en $\mathrm{OD}$ fue de $+13.75-0.50 \times 50^{\circ} 20 / 50$, necesitándose un lente según la fórmula de Mackool de +23.00 dpt. Se implantó el mismo tipo de LIO.

La fórmula de Mackool utilizada es la siguiente ${ }^{10}$ :

Poder de LIO $(\mathrm{dpt})=$ refracción afáquica (equivalente esférico) $\times 1.7$

El paciente mostró buena evolución postoperatoria y no presentó complicaciones. A los 2 meses de la cirugía el paciente presentaba una agudeza visual binocular sin corrección a distancia de 20/25 y una agudeza visual binocular cercana no corregida de J1 en escala de Jaeger. EI OD mostraba una agudeza visual lejana no corregida de 20/25 y cercana no corregida de J3, la agudeza visual lejana corregida fue de 20/25 con $-0.50-0.50 \times 111^{\circ}$. Para el Ol la agudeza visual lejana sin corrección fue de 20/50, cercana sin corrección de $\mathrm{J} 1 \mathrm{y}$ lejana con corrección de 20/20 con-1.00-1.00 × 49 .

\section{Discusión}

Difícilmente podemos determinar de manera acertada cuál es el poder de LIO que se desea utilizar en una cirugía en la que un paciente presenta antecedentes de cirugía refractiva corneal; esto se debe principalmente

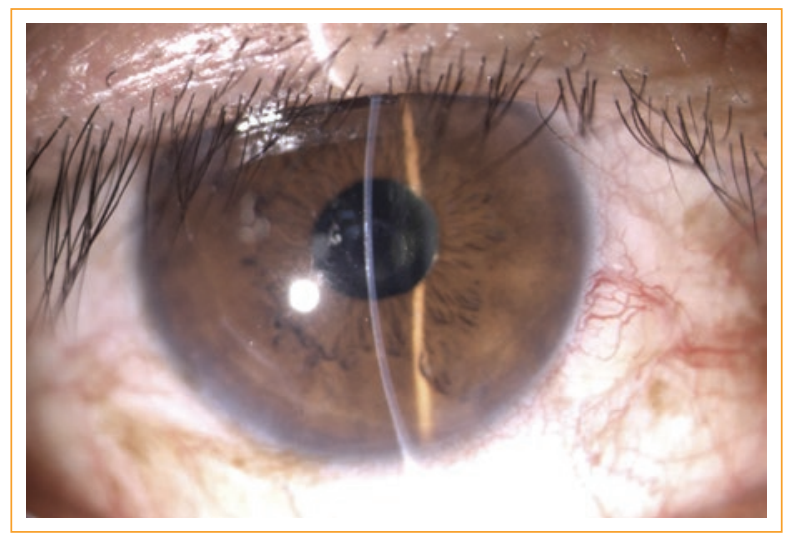

Figura 1. Cicatriz circular en córnea derecha que corresponde al implante Raindrop ${ }^{\circledR}$.

a la modificación de la estructura corneal y a que debemos escoger una fórmula que nos ayude a escoger el LIO correctamente.

Cuando se hallan estas modificaciones en la córnea, se pueden generar mediciones no adecuadas utilizando las herramientas estándar para cálculo de poder de LIO. La cirugía refractiva láser es un antecedente común en la actualidad, y con la probable introducción al mercado de la lentícula Raindrop ${ }^{\circledR}$, esperamos encontrar aún más pacientes que se beneficien de su uso. Mackool et al. presentaron 12 casos de facoemulsificación con antecedente de cirugía LASIK en los que la refracción afáquica rondó entre +8.50 y $+12.375 \mathrm{dpt}$, con un resultado a 2 semanas entre +0.50 y -0.75 dpt. En nuestro paciente, el error refractivo postoperatorio se halló dentro de lo descrito por Mackool et al. ${ }^{10}$. Se esperaba una mejor agudeza visual cercana en el OD gracias al efecto de la lentícula Raindrop ${ }^{\circledR}$, pero aunque el resultado no fue perfecto, es similar al de un paciente con implante de LIO monofocal.

Con una técnica en la que similarmente se obtuvo una refracción afáquica, pero mediante refracción biométrica intraoperatoria con un aberrómetro de frente de onda, lanchulev et al. obtuvieron un error medio de $0.42 \mathrm{dpt}$ en 246 ojos, significativamente mejor que el uso de los métodos de Shammas y Haigis- ${ }^{13}$. Leccisotti en otra técnica de cálculo de LIO en afaquia, en este caso con autorrefracción en ojos ya conocidos como miópicos, obtuvo una media de $0.58 \mathrm{dpt}$ en 82 ojos ${ }^{14}$. En nuestro trabajo, la estimación de poder de LIO más cercana comparada con la técnica de Mackool fue utilizando la fórmula de Shammas junto al resultado de interferometría óptica de coherencia parcial (IOL Master 500) y el paquímetro Scheimpflug (Pentacam). El poder de lente con estos métodos se aleja solo \pm 0.5 
Tabla 1. Comparación entre técnica afáquica de Mackool y las formulas SRK/T y Shammas con diferentes plataformas para cálculo de LIO

\begin{tabular}{|c|c|c|c|c|}
\hline OD & +20 & \multicolumn{2}{|c|}{ 이 } & +23 \\
\hline ES OD & -0.75 & \multicolumn{2}{|c|}{ ES OI } & -1.50 \\
\hline \multirow[t]{2}{*}{ Medición corneal } & \multicolumn{2}{|c|}{ [0,2-3]SRK/T } & \multicolumn{2}{|c|}{$[0,4-5]$ Shammas } \\
\hline & OD & ol & OD & ol \\
\hline$[1,0] \mid 0 L$ Master 500 & $\begin{array}{c}+17 \\
\mathrm{RME}+2.25\end{array}$ & $\begin{array}{c}+19.50 \\
\mathrm{RME}+2\end{array}$ & $\begin{array}{c}+19.50 \\
\text { RME }-0.25\end{array}$ & $\begin{array}{l}+22.50 \\
\mathrm{RME}-1\end{array}$ \\
\hline$[1,0]$ Orbscan II & $\begin{array}{c}+15 \\
\mathrm{RME}+4.25\end{array}$ & $\begin{array}{c}+18.50 \\
\mathrm{RME}+3\end{array}$ & $\begin{array}{c}+17 \\
\mathrm{RME}+2.25\end{array}$ & $\begin{array}{l}+21.50 \\
\text { RME } 0\end{array}$ \\
\hline$[1,0]$ Pentacam & $\begin{array}{c}+18 \\
\mathrm{RME}+1.25\end{array}$ & $\begin{array}{c}+19.50 \\
\mathrm{RME}+2\end{array}$ & $\begin{array}{c}+20.50 \\
\mathrm{RME}-1.20\end{array}$ & $\begin{array}{l}+22.50 \\
\text { RME -1 }\end{array}$ \\
\hline$[1,0]$ Canon RK-F1 & $\begin{array}{c}+18 \\
\mathrm{RME}+1.25\end{array}$ & $\begin{array}{c}+20 \\
\text { RME +1.50 }\end{array}$ & $\begin{array}{c}+21 \\
\text { RME - } 1.75\end{array}$ & $\begin{array}{c}+24 \\
\text { RME }-2.50\end{array}$ \\
\hline
\end{tabular}

ES: equivalente esférico; OD: ojo derecho; OI: ojo izquierdo; RME: refracción manifiesta esperada tras la cirugía. Todos los valores se presentan en dioptrías.

dpt del resultado de la fórmula afáquica de Mackool. Si utilizamos la fórmula SRK/T los resultados se alejan aún más, por encima de $3 \mathrm{dpt}$ en el resultado refractivo final. La agudeza visual lejana binocular sin corrección que presentó el paciente fue $20 / 25$ y cercana J1, con este tipo de desenlace, sería deseable que los resultados de los otros estudios se acerquen lo más posible al de la fórmula de Mackool. Como se muestra en la tabla 1, los resultados pueden llegar a ser catastróficos mientras más nos alejamos de los resultados de la fórmula de Mackool.

\section{Conclusiones}

Para una cirugía de facoemulsificación en ambos ojos en un paciente con antecedente de cirugía LASIK bilateral, retoque e implante de lente intracorneal en OD se utilizó la técnica afáquica de Mackool para calcular el poder de LIO; el desenlace fue aceptable. Creemos que los resultados presentados son de relevancia, no solo por la base de pacientes ya existente en los que se ha realizado cirugía queratorrefractiva, sino porque probablemente en el futuro se comenzará a ver a pacientes con lentículas Raindrop ${ }^{\circledR}$ que necesitarán cirugía de facoemulsificación. Con la descripción de nuestro caso esperamos abrir campo a la discusión del manejo de este tipo de pacientes.

\section{Responsabilidades éticas}

Protección de personas y animales. Los autores declaran que para esta investigación no se han realizado experimentos en seres humanos ni en animales.

Confidencialidad de los datos. Los autores declaran que han seguido los protocolos de su centro de trabajo sobre la publicación de datos de pacientes.

Derecho a la privacidad y consentimiento informado. Los autores declaran que en este artículo no aparecen datos de pacientes.

\section{Financiamiento}

Los autores no recibieron patrocinio para llevar a cabo este artículo.

\section{Conflicto de intereses}

Los autores declaran no tener ningún conflicto de intereses.

\section{Agradecimientos}

Agradecemos a la Consultora en Investigación y Bioestadística "Analimed» la cooperación en la redacción, organización y presentación de este caso. 


\section{Bibliografía}

1. Drexler W, Findl O, Menapace R, et al. Partial coherence interferometry: A novel approach to biometry in cataract surgery. Am J Ophthalmo 1998;126:524-53.

2. Shammas HJ, Shammas MC, Garabet A, et al. Correcting the corneal power measurements for intraocular lens power calculations after myopic laser keratomileusis. Am J Ophthalmol 2003;136:426-32

3 Awwad ST, Manasseh C, Bowman RW, et al. Intraocular lens power calculation after myopic laser insitu keratomileusis: estimating the corneal refractive power. J Cataract Refract Surg 2008;34(7):1070-6.

4. Borasio E, Stevens J, Smith GT. Estimation of true corneal power after keratorefractive surgery in eyes requiring cataract surgery: BESSt formula. J Cataract Refract Surg 2006;32:2004-14.

5. Haigis W. Corneal power after refractive surgery for myopia: contact lens method. J Cataract Refrat Surg 2003;29:1397-411.

6. Norrby S. Pentacam keratometry and IOL power calculation (replica). J Cataract Refract Surg 2008;34:3.

7. Qazi MA, Cual Y, Roberts CJ, et al. Determining corneal power using Orbscan II videokeratography for intraocular lens calculation after excimer laser surgery for myopia. J Cataract Refract Surg 2007;33:21-30.
8. Shammas HJ, Shammas MC. No-history method of intraocular lens power calculation for cataract surgery after myopic laser keratomileusis. J Cataract Refract Surg 2007;33:31-36.

9. Kalyani S, Kim A, Ladas J. Intraocular lens power calculation after corneal refractive surgery. Curr Opin in Ophthalmol. 2008;19:357-62.

10. Mackool RJ, Ko W, Mackool R. Intraocular lens power calculation after laser insitu keratomileusis: Aphakic refraction technique. J Cataract Refract Surg 2006;32:435-43.

11. Whitman J, Dougherty P, Parkhurst G, et al. Treatment of presbyopia in emmetropes using a shape-changing corneal inlay. Ophthalmology. 2016;123:466-75.

12. Steinert R, Schwiegerling J, Lang A, et al. Range of refractive independence and mechanism of action of a corneal shape-changing hydrogel inlay: Results and theory. J Cataract Refract Surg. 2015;41:1568-79.

13. Ianchulev T, Hoffer K, Yoo S, et al. Intraoperative Refractive Biometry for predicting intraocular lens power calculation after prior myopic refractive Surgery. Ophthalmology. 2014;121:56-60.

14. Leccisotti A. Intraocular lens calculation by intraoperative autorefraction in myopic eyes. Graefe's Arch Clin Exp Ophthalmol 2007;246:729-733. 